\title{
Tubulocystic Carcinoma of the Kidney With Multiple Focal Clear Cell Characteristics in a Young Male: A Case Report
}

Shan Zheng ${ }^{1}$, Kao-Peng Guan ${ }^{2}$, Dong Qu ${ }^{3}$, Peng Cao ${ }^{1}$, Chang-Yuan Guo ${ }^{1}$, You-Yan Guan ${ }^{2}$, Chang-ling $\mathrm{Li}^{2}$ and Jian-Hui Ma ${ }^{{ }^{*}}$

${ }^{1}$ Department of Pathology, Cancer Institute and Hospital, Chinese Academy of Medical Sciences and Peking Union Medical College, Beijing, China

${ }^{2}$ Department of Urology, Cancer Institute and Hospital, Chinese Academy of Medical Sciences and Peking Union Medical College,Beijing, China

${ }^{3}$ Department of Imaging diagnosis, Cancer Institute and Hospital, Chinese Academy of Medical Sciences and Peking Union Medical College,Beijing, China

"Corresponding author: Jian-hui Ma, Department of Urology, Cancer Institute and Hospital, Chinese Academy of Medical Sciences and Peking Union Medical College; P.O. Box 2258 Beijing 100021, P.R.China. Tel: 86-10-8778 8500; Fax: 86-10-87715889; E-mail: majianhui@csco.org.cn

Received date: Nov 24, 2014, Accepted date: Jan 21, 2015, Published date: Jan 25, 2015

Copyright: (c) 2015 Zheng S, et al. This is an open-access article distributed under the terms of the Creative Commons Attribution License, which permits unrestricted use, distribution, and reproduction in any medium, provided the original author and source are credited.

\begin{abstract}
Tubulocystic carcinoma of the kidney is a rare entity, which are with about 100 cases of these tumors of the kidney all over the world. We have reported a case, of a 43-year-old man who presented with a lump in the right kidney on image examination without other complaints. Final pathologic diagnosis was tubulocystic carcinoma of the kidney with multiple focal clear cells. Six months after the procedure, the patient remained free of disease. This case showed an indolent manner a low-grade malignancy with favorable prognosis in a short-time follow-up.
\end{abstract}

Keywords: Tubulocystic carcinoma; KidneyAbbreviation: AMACR: Alpha-Methylacyl-CoA Racemase; TC: Tubulocystic Carcinoma; MRI: Magnetic Resonance Imaging; WHO: World Health Organization

\section{Introduction}

Tubulocystic carcinoma (TC) of the kidney is a rare tumor which may be recommended to list in the next edition of World Health Organization (WHO) classification in kidney tumor [1]. The clinical, pathological, immunohistochemistrical characters of this tumor were first summarized systematically by MacLennan and his colleagues $[2,3]$. Now, there were about 100 cases of TC of the kidney all over the world. Here, we reported a case of TC with multiple focal clear cells in China.

\section{Case Presentation}

A 43-year-old male presented with a lump in the right kidney on Magnetic Resonance Imaging (MRI) examination without other complaints. His past medical history and family history was unremarkable. The patient's physical exam was negative. A $1.5 \mathrm{~cm}$, solid, non-enhancing mass located in the outside of the upper pole in the right kidney in Magnetic Resonance Imaging (MRI) (Figure 1). Additional workup for metastatic disease was negative. Preoperative diagnosis considered that the mass might be angiomyolipoma or and small renal cell carcinoma were all possible. The patient was underwent partial nephrectomy. No adjuvant therapy was administered to the patient. Six months after the procedure, the patient remained free of disease.

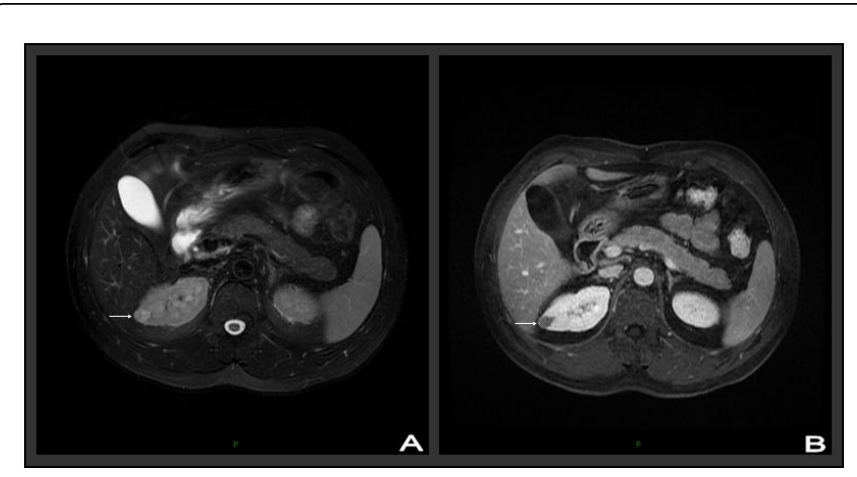

Figure 1: Magnetic Resonance Imaging showed a small mass in the outside of the upper pole in the right kidney. A, Fat Suppression T2-weighted imaging showed a high signal mass measured 1.5$\times 1.4-\mathrm{cm}$, which was not homogeneous inside the mass. B, Enhancement showed the mass was not enhanced.

\section{Gross characteristics}

A wedge specimen of the kidney of $2.4-\times 2.0-\times 1.5 \mathrm{~cm}$ was examined sent to the department of pathology. The specimen size was $2.4 \times 2.0 \times 1.5 \mathrm{~cm}$. The cut surface showed an ill-circumscribed solid tumor. The size of the tumor was $1.5-\times 1.5-\times 1.0 \mathrm{~cm}$. The tumor involved the renal capsule.

\section{Microscopic Characteristics}

The tumors were composed of closely packed small tubules and cysts, with some slender papilla pattern (Figure 2A). The tumor grew into the surrounding normal tissue (Fig 2B). These tubules and cysts were separated by thin fibrous septa without an ovarian-type of stroma or desmoplastic reaction (Figure 2C). The epithelial cells lining the tubules and cysts were simple flat, cuboidal or hobnail cell. The 
cytoplasm of most cells was eosinophilic, while some cells showed clear cytoplasm (Figure 2C-2E). The lining cells showed prominent nucleoli equivalent to that of Fuhrman nuclear grade 3 (Figure 2F). There was no solid sheets growth pattern in the tumor.

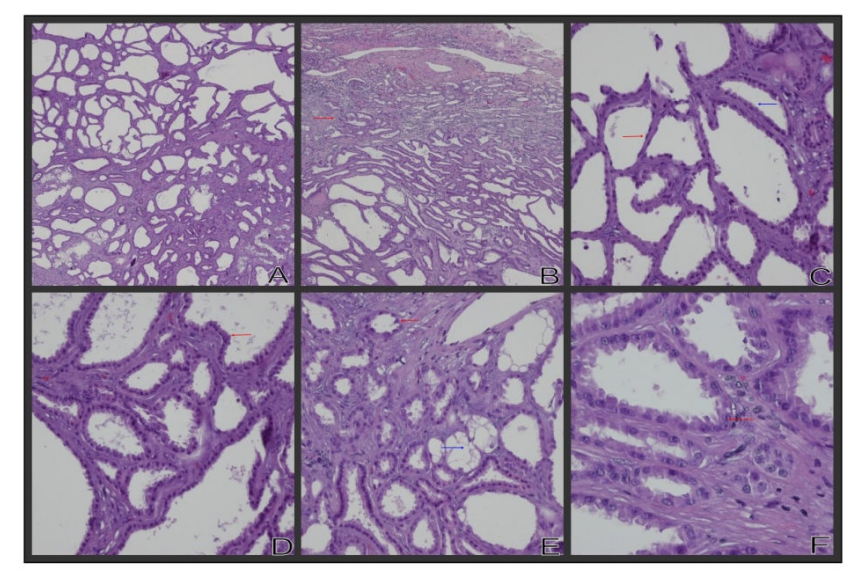

Figure 2: The tumor is composed of small tubules and cysts, with some slender papilla pattern (A) and the boundary between tumor and normal tissue is ill-defined invasive. The red arrow showed the ill tumor's boundary (B). Tumor cells are flat (red arrow) and cuboidal (blue arrow) with thin fibrous septa (C). Tumor cells also show high column (D, red arrow), hobnail cell (E, red arrow) or clear cytoplasm (E, blue arrow). Some tumor cells show prominent nucleoli (F, red arrow).

\section{Immunohistochemical Features}

Immunohistochemical results showed that the tumor cells were strong positive immunoreactive for the presence of CK8, CK18, CK19, CD10 and PAX8. The tumor cells were also showed positive stains associated with for alpha-methylacyl-CoA racemase (AMACR) and Vimentin, while the stains of CK7, CK34ßE12, PAX2 and P53 were was focal or weak. The tumor cells showed a low Ki-67 index (Figure 3).

\section{Discussion}

We report the characteristic of a case of TC with multiple focal clear cells in China mainly focused on pathology.

Srigley et al. thought that TC was originally named "Bellinian epithelioma" in 1956 [1]. In 1997, MacLennan et al. reported 13 cases of unusual low-grade renal carcinomas with features most suggestive of collecting duct origin [2]. The latter research found that these 13 cases included two different type of renal cell carcinoma-TC and mucinous tubular and spindle cell carcinoma [3]. The latter was listed into the WHO's classification of tumors of the urinary system and male genital organs in 2004, while the former was proposed as new epithelial neoplasms in next edition of WHO classification $[1,4,5]$.

Most patients of TC are adults with a male predominance, while the peak age range of diagnosis is in the fifth and sixth decades [6-8]. Most patients are asymptomatic, and the majority of these patients come to the hospital with the reveal of a mass in kidney in imaging examination test $[7,9,10]$.

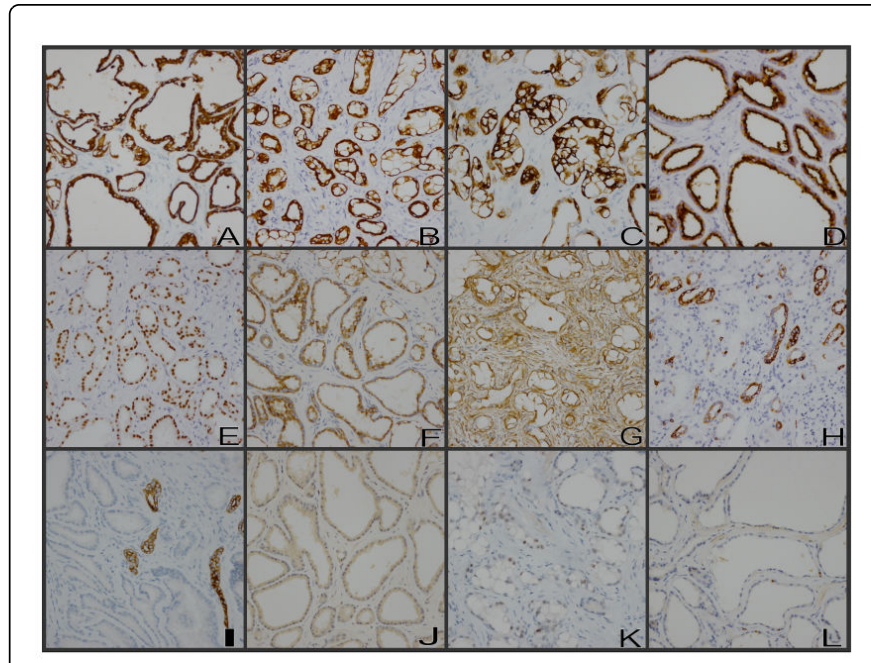

Figure 3: The immunohistochemistry characters of tubulocystic carcinoma of the kidney. Note the strong diffuse expression of CK8 (A), CK18 (B), CK19 (C), CD10 (D), PAX8 (E); and the middle diffuse expression of AMACR (F), Vimentin (G); and the strong focal expression of CK7 (H), CK34ßE12 (I); and the weak or focal expression of PAX2 (J), P53 (K) in neoplastic cells. The Ki-67 index was very low $(<5 \%)$ in neoplastic cells $(\mathrm{L})$.

Macroscopically, the tumors are well circumscribed usually, with a "bubble wrap" or "Swiss cheese" appearcance. The maximum size was no more than $5.0 \mathrm{~cm}$ in most cases [7,8,10-14]. Microscopically, the tumors are composed of closely packed small tubules and cysts. There are thin fibrous septa separating these tubules and cysts. The tumor cells are simple flat, cuboidal or hobnail cell. The cytoplasm is eosinophilic in most cells, while some cells may show clear cytoplasm. The prominent nucleoli may be one of the important characters in these tumors [6-8,10,12-14]. Immunohistochemically, the tumors share the features of both proximal (CD10 and AMACR) and distal tubules (CK19) [10,11]. Here, we reported a case of TC in a young patient. The gross specimen showed an ill-circumscribed solid tumor, which was atypical for TC. The characters of gross reminded us of angiomyolipoma or small renal cell carcinoma. Howerver, the histopathologic characters supported the diagnosis. The main differential diagnosis in this case was multilocular cystic renal cell carcinoma as this case showed multiple focal clear cells. The characters of nuclear helped us to make the diagnosis. In multilocular cystic renal cell carcinoma, the nuclear showed Fuhrman nuclear grade 1. In TC, the nuclear showed obvious nucleus which showed Fuhrman nuclear grade 3. The significance of the clear cells was not clear. Our case was according with the description above. However, we found some clear cytoplasm cells in our case. As we had a short time follow-up, the significance of these cells was not clear. It needs a long follow-up time to answer this question.

In summary, TC is a new entity of renal cell carcinoma with a distinct macroscopical, microscopical and immunohistochemical characters. The origin of the tumor is not clear. The early researches thought it was a subtype of collecting duct carcinoma CDC, while the recent study considered it may be related to the papillary renal cell carcinoma $[2,6]$. This tumor should be distinguished from other cystic diseases of the kidney. There is no treatment guideline for this rare 
Citation: Zheng S, Guan KG, Qu D, Cao P, Guo CY, et al. (2015) Tubulocystic Carcinoma of the Kidney With Multiple Focal Clear Cell Characteristics in a Young Male: A Case Report. J Carcinog Mutagen 6: 213. doi:10.4172/2157-2518.1000213

Page 3 of 3

tumor. The thorough comprehension of the molecular mechanism in this tumor may help the treatment and management.

\section{References}

1. Srigley JR, Delahunt B, Eble JN (2013) The International Society of Urological Pathology (ISUP) Vancouver Classification of Renal Neoplasia. Am J Surg Pathol 37: 1469-1489.

2. MacLennan GT, Farrow GM, Bostwick DG (1997) Low-grade collecting duct carcinoma of the kidney: report of 13 cases of low-grade mucinous tubulocystic renal carcinoma of possible collecting duct origin. Urology 50: 679-684.

3. MacLennan GT, Bostwick DG (2005) Tubulocystic carcinoma, mucinous tubular and spindle cell carcinoma, and other recently described rare renal tumors. Clin Lab Med 25: 393-416.

4. Murphy WM, Beckwith JB, Farrow GM (1994) Atlas of Tumor Pathology Fascicle 11, Tumors of the Kidney, Bladder and Related Urinary Structures. 3rd ed. Washington, DC: Armed Forces Institute of Pathology

5. Eble JN, Sauter G, Epstein JI (2004) World Health Organization Classification of Tumours. Pathology and Genetics of Tumours of the Urinary System and Male Genital Organs. Lyon, France: IARC; 2004.

6. Yang XJ, Zhou M, Hes O (2008) Tubulocystic carcinoma of the kidney: clinicopathologic and molecular characterization. Am J Surg Pathol 32: 177-1787.
7. Amin MB, MacLennan GT, Gupta R (2009) Tubulocystic carcinoma of the kidney: clinicopathologic analysis of 31 cases of a distinctive rare subtype of renal cell carcinoma. Am J Surg Pathol 33: 384-392.

8. Alexiev BA, Drachenberg CB (2013)Tubulocystic carcinoma of the kidney: a histologic, immunohistochemical, and ultrastructural study. Virchows Arch 462(5): 575-581.

9. Brennan C, Srigley JR, Whelan C, Cooper J, Delahunt B (2010) Type 2 and clear cell papillary renal cell carcinoma, and tubulocystic carcinoma: a unifying concept. Anticancer Res 30: 641-644.

10. Moses KA, Decaro JJ, Osunkoya AO, Issa MM (2010) Tubulocystic carcinoma of the kidney: a case report of natural history and long-term follow-up. ScientificWorldJournal 10: 586-589.

11. Azoulay S, Vieillefond A, Paraf F (2007) Tubulocystic carcinoma of the kidney: a new entity among renal tumors. Virchows Arch. 451: 905-909.

12. Chen N, Nie L, Gong J (2014) Gains of chromosomes 7 and 17 in tubulocystic carcinoma of kidney: two cases with fluorescence in situ hybridisation analysis. J Clin Pathol

13. Iakovleva G, Iakovlev V, Ordon M, Srigley J, Yousef GM (2014) Tubulocystic Carcinoma of Kidney: a Challenging Diagnostic Entity Mimicking Multicystic Kidney and Presenting with Bone Metastasis. Histopathology

14. Ishibashi Y, Koie T, Fujita N (2014) Tubulocystic renal cell carcinoma in the left kidney: a case report. J Med Case Rep 8: 265. 